\title{
Neue berufliche Chancen im Kampf gegen den Nachwuchsmangel
}

\section{Marianne Schenk ${ }^{a}$, Carlos Beat Quintob}

a Präsidentin Odamed Berufsbildung Medizinische Praxisassistentin, ${ }^{\mathrm{b}}$ Dr. med., Mitglied des FMH-Zentralvorstandes, Departementsverantwortlicher Public Health und Gesundheitsberufe

Die Tätigkeiten einer Medizinischen Praxisassistentin (MPA) sind interessant und attraktiv. Leider besteht aber auf dem Arbeitsmarkt immer noch ein genereller MPA-Mangel und es müssen Massnahmen getroffen werden, diesen zu beheben. Die Weiterbildung zur Medizinischen Praxiskoordinatorin (MPK) eröffnet den MPA nun neue berufliche Perspektiven und steigert damit die Attraktivität des Berufs. Diese Tatsache und der Umstand, dass dadurch die Anzahl Lernende gegenüber dem letzten Jahr leicht zugenommen hat, reichen aber alleine nicht aus. Es müssen weitere Anstrengungen unternommen werden, um den Nachwuchs in diesem Beruf weiter zu fördern. Lernende auszubilden bedeutet für den Betrieb immer Mehraufwand und Investition. Dank dem Engagement von MPA und Arzt gelingt dies fortan mit guter Qualität.

Die Hürden sind für die Lehrbetriebe in der Selektion von Lernenden relativ hoch, auch weil die Berufslehre immer noch ein «Image-Problem» hat. Der Trend nach

Seit dem 1. August 2017 werden Absolventinnen und Absolventen von vorbereitenden Kursen für die eidgenössische Berufsprüfung und höhere Fachprüfung vom Bund finanziell unterstützt. So auch Medizinische Praxisassistentinnen bzw. -assistenten MPA EFZ, die sich zur Medizinischen Praxiskoordinatorin bzw. zum Praxiskoordinator MPK weiterbilden und einen Vorbereitungskurs besuchen. Wichtig ist, dass die MPA die Kurskosten selber in ihrem Namen bezahlen und der Arbeitgeber seinen Anteil an die Kurskosten danach mit den MPA direkt abrechnet. Ansonsten haben die MPA kein Anrecht auf eine Rückerstattung der Kurskosten.

Genauere Informationen für praktizierende Ärztinnen und Ärzte, die MPA beschäftigen, die sich zur MPK weiterbilden, finden Sie auf der FMH-Website unter: http://www.fmh.ch/ $\rightarrow$ Services $\rightarrow$ Medizinische Praxisassistentinnen $\rightarrow$ MPA EFZ: Bundesbeiträge zu Vorbereitungskursen Berufsprüfung MPK (rechts in der dritten grauen Box) höheren Berufsausbildungen und den damit verbundenen besser bezahlten Jobs trägt das Seinige bei. Auf potentielle Lehrstellen bewerben sich nicht selten ungenügend qualifizierte Jugendliche. Die idealen Ausbildungsplätze für lernende MPA sind die Hausarztpraxen, weil diese in der Regel alle Kernkompetenzen einer MPA für die Berufsausbildung anbieten können. Spezialarztpraxen, welche nicht das ganze Kompetenzspektrum in ihrem Praxisalltag anbieten können, können sich in Verbund- bzw. Kooperationslösungen als Ausbildungspraxen etablieren. Zudem sind die Spitäler auf den Geschmack gekommen, MPA anzustellen und zeigen sich zunehmend auch bereit, MPA auszubilden, auf einen gewissen Druck hin und aus Gründen der Fairness gegenüber der Praxisärzteschaft und den kantonalen Ärztegesellschaften, welche die Ausbildung personell und finanziell tragen. Die MPA finden zunehmend Arbeitsstellen in den Ambulatorien von Spitälern. Der Mangel an Lehrstellen steht auch - aber nicht nur - im direkten Zusammenhang mit dem Rückgang der Hausärzte. Das erfordert Massnahmen für mehr Lehrstellen und Alternativen, um den Nachwuchs zu sichern, sowie Massnahmen für die Qualität, um den Anforderungen dieser anspruchsvollen Berufslehre gerecht zu werden. 


\section{Anforderungen an Lernende}

Nicht unerheblich ist dabei der Selektionsprozess von Lernenden. Mit der richtigen Auswahl von Lernenden entscheidet sich weitgehend, wie aufwändig deren Begleitung am Arbeitsplatz sein wird. Wir plädieren deshalb auf eine gute Vorbildung der Jugendlichen auf einem Schulniveau der erweiterten Sekundarstufe 1 und guten Noten in den naturwissenschaftlichen Fächern. Die Ausbildung zur MPA ist vielseitig und erfordert ein hohes Mass an Engagement in Schule und Betrieb. Der duale Ausbildungsweg schafft die Grundlage, gelerntes Wissen in die Praxis umzusetzen. Die Fähigkeit zu vernetzen sehen wir als eine der wichtigsten Anforderung an die Lernenden! Die Handlungen stehen im Fokus und die Fähigkeit, benötigtes Wissen selbst zu produzieren. Die MPA-Ausbildung ist ein enges Zusammenspiel zwischen Lernen und Arbeiten. Vernetztes Denken kann nur entwickeln, wer über ein genügend breites Wissen verfügt. Genau deshalb ist es wichtig, den Lernenden gute und gesicherte Fertigkeiten und das aktuellste Wissen anzubieten und sie darin zu unterstützen, selbstverantwortlich für den eigenen Lernerfolg zu handeln. Ist die MPA ausgebildet, ist ihre Persönlichkeit gereift und der Umgang mit Menschen gestärkt und geformt. Dies macht die jungen Berufsleute zu charakterstarken, hilfsbereiten und sozialen Mitmenschen, die sich für die Arztpraxis weiterbilden und -entwickeln können. Sie werden so stets einen guten und sicheren Stellenwert geniessen und mit entsprechender Förderung und Unterstützung durch die Ärzte auch längerfristiger bindend dem Betrieb erhalten bleiben.

\section{Attraktivität steigern und Lehrstellen fördern}

Einige Massnahmen zur Förderung von Lehrstellen sind in Planung und werden in naher Zukunft umgesetzt. So wird eine Promotion des MPA-Berufs an den nationalen Berufsmeisterschaften SwissSkills durchgeführt werden, welche auf dem Expo-Gelände in Bern vom 13. bis 16. September 2018 stattfinden wird. Mit einem gemeinsamen Informationsstand wollen die FMH und der SVA, unter der Beteiligung weiterer Organisationen wie mfe, JHAS und die ARAM, einen Einblick in den MPA-Beruf geben. Weiter konnte im Rahmen der BiVo-Revision 2019 die Attraktivität für Lernende gesteigert werden, denn diese werden $a b$ dann einen halben Tag mehr in der Arztpraxis tätig sein. Mit dem "Strategiepapier MPA» wird von der FMH und den MPA-Berufsverbänden eine zukunftsgerichtete Publikation geschaffen, welche als Grundlage und Orientierung bei der Umsetzung der einzelnen Massnahmen dienen soll.

\section{Neue Weiterbildungsmöglichkeit}

Die Entwicklung der Berufsprüfung zur Medizinischen Praxiskoordinatorin und die gut besuchten SchulModule zeigen, dass ein Schritt in die richtige Richtung getan ist. Wir sind zuversichtlich, dass die MPA mit dieser Weiterbildungsmöglichkeit vermehrt im Beruf bleiben werden und weniger in andere Berufszweige abwandern.

Für Ärztinnen und Ärzte können die neuen Berufsleute gute Unterstützung im Praxisalltag bieten. Das digitale Zeitalter ist mit grossen Veränderungen für die Arztpraxen schon längst eingetreten und schreitet weiter voran. Die Prozesse sind beschleunigt, vielfach effizienter, aber keinesfalls kostengünstiger! Die MPK wird ihre Rolle in diesem Bereich wahrnehmen und Ärztinnen und Ärzte in ihren Unternehmen entlasten können.

Die beiden Fachrichtungen geben vor, in welchen $\mathrm{Ge}$ bieten die MPK zum Einsatz kommen: Die MPK praxisleitende Richtung verbinden klinisch-medizinische Erfahrung mit Geschäftsführungs-Know-how. Sie arbeiten in einer Kaderfunktion und sind in einer Arztpraxis mit dem selbständigen Führen des Personals, des HR- und Einkommensmanagements, des Praxismarketings und der Qualitätssicherung betraut.

Die MPK klinischer Richtung arbeiten und betreuen unter der Verantwortung einer Ärztin oder eines Arztes Menschen mit einer oder mehreren chronischen Krankheiten. Sie sind besorgt um eine patientenzentrierte Koordination von Abklärungen und Therapien der involvierten Leistungserbringer und erleichtern den Zugang zu den kommunalen Ressourcen. Sie kennen die wichtigsten Aufgaben und Handlungskompetenzen der Fachpersonen im Gesundheitswesen und respektieren ihre eigenen fachlichen und beruflichen Grenzen.

\section{Effizienzsteigerung in den betrieblichen Abläufen}

Das Arbeitsumfeld in der ambulanten medizinischen Versorgung ist von einer immer grösser werdenden Lücke zwischen nachgefragten und angebotenen Konsultationen bei den Hausärztinnen und Hausärzten und der Zunahme älterer Menschen mit komplexen chronischen Krankheiten geprägt. Der dadurch entstehenden Überlastung der Arztpraxen kann durch den Einbezug einer MPK in verantwortungsvolle Tätigkei- 
Korrespondenz: OdA Berufsbildung Medizinische Praxisassistentin

Elfenstrasse 19 Postfach 1010

CH-3000 Bern 6

Tel. 0313805458

Fax 0313810457

info@odamed.ch ten im Rahmen eines Teamansatzes begegnet werden. Die MPK leisten ihren Beitrag an die Gesellschaft im Rahmen einer kosteneffizienten Gesundheitsversorgung, im klinischen Bereich durch Beratung und Behandlung von Langzeitpatienten, im administrativen Praxisbereich zu Gunsten eines wirksamen Personal-, Kosten- und Qualitätsmanagements.

Für die Praxisinhaber bedeutet dies einerseits eine Entlastung in der Betriebsorganisation zugunsten ihrer Kernaufgaben in der ambulanten Medizin und andererseits organisatorische Entlastung in der Betreuung von Menschen mit chronischen Krankheiten. Die ausgebildeten MPK tragen damit zur Effizienzsteigerung in den betrieblichen Abläufen der Arztpraxis bei und helfen, dass in der kostengünstigeren Praxis ausreichend Kapazität für die Betreuung von chronisch kranken Patienten vorhanden ist und diese nicht in den teureren spitalambulanten Bereich ausweichen müssen.

\section{Moderne und vernetzte Arztpraxen}

Mit dem enormen technischen Fortschritt und den zunehmenden gesellschaftlichen Ansprüchen an die ärztliche Versorgung sehen sich traditionelle Praxismodelle mit zahlreichen Herausforderungen konfrontiert. Die neuen Weiterbildungsmöglichkeiten der MPA bieten einen Weg, auf diese Herausforderungen $\mathrm{zu}$ reagieren. In Kombination mit alternativen Betriebsmodellen wie Gemeinschaftspraxen, «fliegenden» Dienstleistern und/oder Praxisnetzwerken kann die MPK dazu beitragen, die Hausarztpraxis zu einem modernen und vernetzten Betrieb werden zu lassen. Nun ist es Sache der medizinischen Leistungserbringer nicht hinterher zu hinken, damit die Bevölkerung auch zukünftig vertrauensvoll auf die Kompetenz und die Kommunikationsfähigkeit ihrer Hausarztpraxen setzen kann. 